\title{
Activatable Photosensitizers: Agents for Selective Photodynamic Therapy
}

\author{
Xingshu Li, Safacan Kolemen, Juyoung Yoon,* and Engin U. Akkaya*
}

Recent developments in the design of bifunctional and activatable photosensitizers rejuvenate the aging field of photodynamic sensitization and photodynamic therapy. While systematic studies have uncovered new dyes that can serve as potential photosensitizers, the most promising results have come from studies aimed at gaining precise control over the location and rate of cytotoxic singlet oxygen generation. As a consequence, higher selectivities and efficiencies in photodynamic treatment protocols are now within reach. This feature article highlights the variety of approaches that have been pursued to improve photodynamic therapy and to transform simple photosensitizers into smarter theranostic agents.

tendencies to accumulate in tumor tissues to a greater extent than they do in normal tissues. On the other hand, irradiation light (in the red or near IR region) used for exciting the PS can be tightly focused on the tumor regions, essentially assuring the selective generation of singlet oxygen. Moreover, some other advantages of PDT, such as enhancement in the immune response ${ }^{[2]}$ and minimally invasive treatment methods, ${ }^{[3]}$ make PDT very attractive in clinical applications compared to conventional therapies.

Earlier PSs employed in PDT were based on porphyrins. In fact, the first PS-

\section{Introduction}

Photodynamic action, a term that was introduced at the turn of the $20^{\text {th }}$ century, is at the core of photodynamic therapy (PDT). ${ }^{[1]}$ Upon light irradiation, certain dyes (i.e., photosensitizers, PS) undergo intersystem crossing (ISC) and rapidly form their triplet excited states. The triplet-excited state energy is then transferred to ground state (triplet) molecular oxygen (Figure 1) to generate singlet-excited state of oxygen (singlet oxygen). The half-life of singlet oxygen in aqueous milieu is about $1 \mathrm{~ms}$ and it is cytotoxic as a result of its high reactivity towards several vital biomolecules Thus, PDT is a promising cancer treatment modality in which the therapeutic action can be satisfied by generating singlet oxygen in tumors regions. One important contribution to the critically needed tumor selectivity of PDT is associated with the fact that PSs, which can be designed and constructed with targeting groups (active targeting strategy) or nano-delivery systems (passive targeting strategy), have

\section{Dr. X. Li, Prof. J. Yoon}

Department of Chemistry and Nano Science

Ewha Womans University

Seoul 120-750, Korea

E-mail:jyoon@ewha.ac.kr

Dr. S. Kolemen, Prof. Dr. E. U. Akkaya

UNAM-Institute of Material Science

and Nanotechnology

Bilkent University

Ankara 06800, Turkey

E-mail: eua@fen.bilkent.edu.tr

Prof. Dr. E. U. Akkaya

Department of Chemistry

Bilkent University

Ankara 06800, Turkey

DOI: 10.1002/adfm.201604053 approved by the FDA in 1995 for PDT of certain types of cancers was hematoporphyrin, an endogenous porphyrin obtained by acidic hydrolysis of hemoglobin. Within the last two decades, highly diverse types of dyes have been studied as potential PSs for PDT. Fluorescence emission and intersystem crossing to the triplet manifold of a PS are two major photophysical processes that take place after forming the singlet excited states of PSs. Owing to this factor, fluorescence emission competes with ISC and following singlet oxygen generation processes. However, these outcomes are not mutually exclusive (Figure 1). Thus, it is possible for a PS to participate in high yield of ISC and singlet oxygen production while at the same time having a remarakable fluorescence quantum yield. As a result, PSs can serve as theranostic agents by displaying the dual functions of fluorescence imaging and photodynamic activity (vide infra).

The focus of this feature article is on new PSs, termed activatable photosensitizers (aPSs), which remain in a passive state even under light irradiation. However, aPSs are activated at the site where therapy is desired by tumor-associated stimuli. ${ }^{[4,5]}$ Since aPSs can have their PDT and tumor imaging capabilities at the same time, they rejuvenate the field of PDT by making it highly possible to reduce the side effects and unselective killing of healthy cells. Below, we present a current summary of the progress that has been made in the development of aPSs as phototheranostic agents to assess tumor microenvironments.

\section{Design of aPSs}

\subsection{A Palette of Dyes}

Porphyrin-based substances, whose structures contain four pyrrole rings conjugated by methine bridges in a cyclic configuration (Figure 2), are the most common PSs used in 


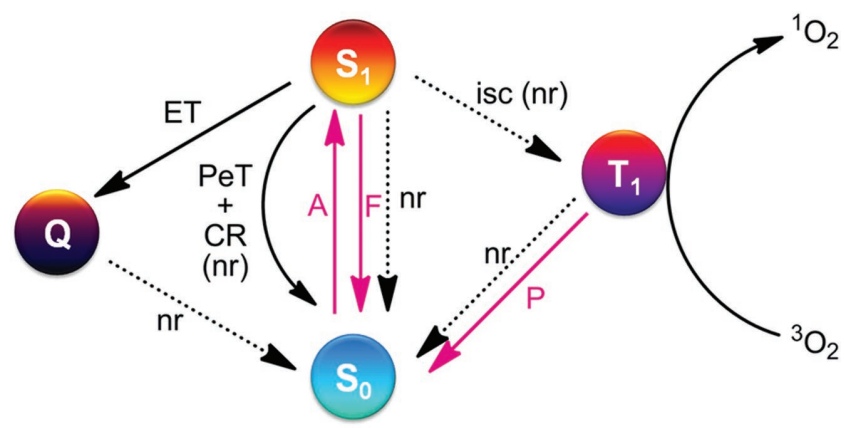

Figure 1. Relevant photophysical events involved in photosensitized formation of singlet molecular oxygen, including photodynamic action. Red arrows denote photonic and black arrows (solid or dashed) denote non-radiative $(\mathrm{nr})$ processes. A: absorption, F: fluorescence, $\mathrm{P}$ : phosphorescence, $\mathrm{ET}$ : energy transfer, $\mathrm{PET}$ : photoinduced electron transfer, $\mathrm{CR}$ : charge recombination, ISC: intersystem crossing.

PDT. Because of their $18 \pi$-electron aromatic macrocyclic structures, porphyrins typically have characteristic electronic absorption spectra with characteristic Q bands in the 500-700 nm region and a Soret band around $400 \mathrm{~nm} \cdot{ }^{[6,7]}$ With appropriate modification, the basic structure can be transformed into porphyrin derivatives or other porphyrinoids that have specific photoactivities. For example, chlorin is a reduced form of porphyrin, which is obtained by reduction of one the peripheral cross-conjugated bond. ${ }^{[8]}$ Owing to the change in symmetry that is brought by reduction, the Q-bands in chlorins are red shifted and have large extinction coefficients. ${ }^{[8]}$

Phthalocyanines (Pcs), on the other hand, are related to tetraazaporphyrins in that they possess four Schiff base rather than methane bridges connecting the pyrrole rings (Figure 2). Since their discovery in 1907 by Brown and Tcheriac, ${ }^{[9]}$ Pcs have been subjected to numerous studies focusing on ways to optimize their photophysical and photochemical properties. ${ }^{[10,11]}$ Pcs are well suited as PSs for bioimaging and PDT because of their tunable photophysical properties and more efficient generation of singlet oxygen compared to those of porphyrins. ${ }^{[12,13]}$ Because they have an extended conjugated electronic structure, Pcs exhibit enhanced absorption at wavelengths around $670 \mathrm{~nm}\left(\varepsilon \approx 10^{5} \mathrm{~L} \mathrm{~mol}^{-1} \mathrm{~cm}^{-1}\right)$, which are longer than those of most porphyrins $\left(\lambda_{\max } \approx 610 \mathrm{~nm}, \varepsilon \approx 5 \times 10^{3} \mathrm{~L} \mathrm{~mol}^{-1} \mathrm{~cm}^{-1}\right.$ )
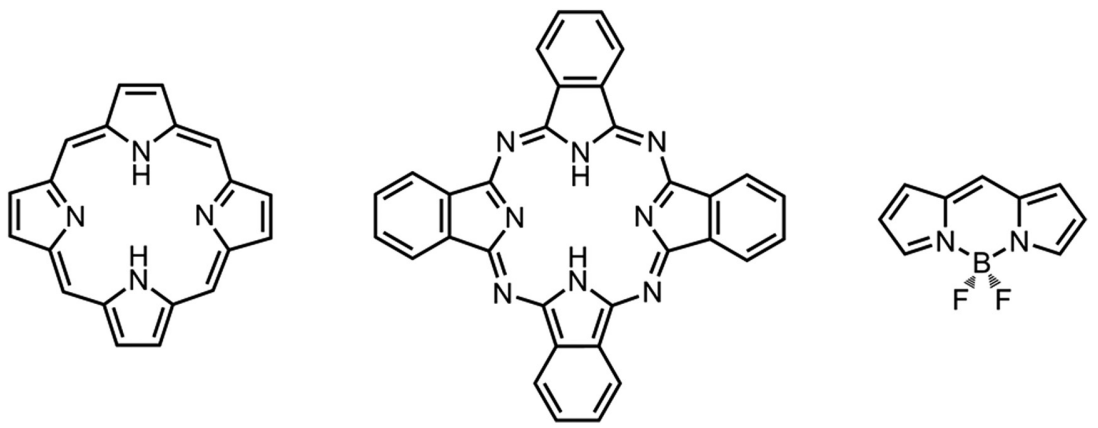

Figure 2. Parent structures of the most commonly used dyes in activatable PS design. Left: porphin, middle: phthalocyanine (Pc), right: BODIPY. BODIPY in the above form, absorbs near $500 \mathrm{~nm}$, but straightforward derivatizations push the absorption peak well into the red and near IR region.

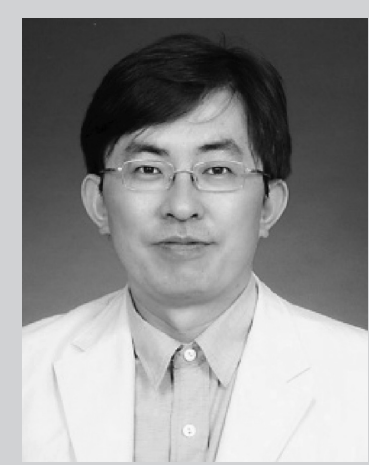

Prof. Juyoung Yoon received his Ph.D. (1994) from The Ohio State University. After completing postdoctoral research at UCLA and at Scripps Research Institute, he joined the faculty at Silla University in 1998. In 2002, he moved to Ewha Womans University, where he is currently a Distinguished Professor of Department of Chemistry and Nano Science.

His research interests include investigations of fluorescent chemosensors, molecular recognition, and organic functional materials.

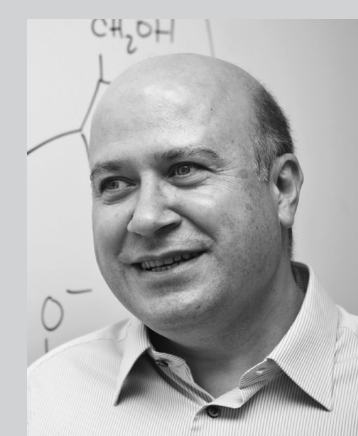

Prof. Engin U. Akkaya (B.Sc METU; M.S. and Ph.D. The Ohio State University) is in the Department of Chemistry and UNAM-National Nanotechnology Research Center, Bilkent University, Ankara, Turkey. His current research interests are photodynamics, molecular logic gates and molecular devices, information processing therapeutic agents, and photochemical control of biological processes.

and that penetrate deeper into biological tissues. ${ }^{[14]}$ Specificity required for the applications of Pcs can be governed by modification of the peripheral aromatic rings, by the nature of central chelated metal ions and even by altering the axial ligands. Pcs that have been probed as PSs for PDT include derivatives of zinc(II)-phthalocyanine (ZnPc), silicon(IV)-phthalocyanine (SiPc), and aluminum(III)-phthalocyanine (AlPc). ${ }^{[15]}$ In recent years, many ZnPc- and SiPc-based PSs have been developed for potential applications in near-infrared fluorescence imaging and PDT.

BODIPY dyes are relatively recent additions to the PS arsenal (Figure 2). These substances have remarkable fluorescence properties, which make them highly attractive for bioimaging applications. ${ }^{[16-18]}$ It is also possible to alter the excited state dynamics of BODIPYs by suitable structural modifications in such a way that effective ISC and consequential singlet oxygen generation become more efficient. One method of enhancing ISC involves introduction of heavy atoms (eg., bromine and iodine) onto the BODIPY core. ${ }^{[19,20]}$ Efficient singlet oxygen generation along with other prominent characteristics, such as tunable excitation wavelengths, high extinction coefficients, rapid cellular uptake, significant 
photostability and ease of functionalization, make BODIPY derivatives popular PSs in PDT applications. ${ }^{[19,20]}$ Recently, a new generation of BODIPY-based PSs has been developed that undergo efficient ISC and PDT action without requiring the presence of heavy atom substituents on the core structure. [21]

\subsection{Low $\mathrm{pH}$ of Tumor Microenvironments Activates Cancer-Specific Imaging and PDT}

Tumor tissues have unique microenvironments that are slightly acidic, as reflected in the fact that the extracellular $\mathrm{pH}$ in these tissues is usually lower (pH 6.5 to 7.2) than that in normal tissues (pH 7.4). ${ }^{[22]}$ This phenomenon is a consequence of the greater activity of glycolysis and plasma membrane protonpumps in cancer cells, which elevate the generation of lactic acid. ${ }^{[23]}$ Therefore, the utilization of PSs that are highly fluorescent and promote singlet oxygen production at acidic $\mathrm{pHs}$, but are less active at $\mathrm{pH}$ ca. 7.4, would facilitate cancer-specific imaging and PDT.

By using this reasoning, Ju et al. designed novel targeted PSs, which contain a $\mathrm{pH}$-activatable theranostic nanoplatform (cRGD-NEt $\mathrm{Br}_{2} \mathrm{BDP} \mathrm{NP}$ ), by encapsulating diethylamino-phenyl and -bromophenyl substituted aza-BODIPYs (Figure 3a) into a cyclic RDG (cRDG) peptide-poly(ethylene glycol)-block-poly(lactic acid) micelle. ${ }^{[24]}$ The cRDG nanomicelle shells of these PSs ensures that they target $\alpha_{\mathrm{v}} \beta_{3}$ integrin-rich cancer cells. In accord with the design features, these PSs undergo both singlet oxygen generation $\left(\Phi_{\Delta}=56 \%\right)$ and NIR fluorescence emission at $925 \mathrm{~nm}$ selectively under slightly acidic conditions $(\mathrm{pH}<7.0)$. At these $\mathrm{pHs}$, protonation of dimethylaminoaryl groups takes place to block photo-induced electron transfer (PET) from the amine donor to the excited BODIPY chromophore. Importantly, implementation of one of these PSs, aza-BODIPY-based $\mathrm{NEt}_{2} \mathrm{Br}_{2} \mathrm{BDP}$ (Figure 3), enables selective detection of NIR fluorescence emission only around tumor regions in a U87MG tumor-bearing mouse.

The important features of this imaging system are that it displays a high tumor-to-background ratio (10-fold, $8 \mathrm{~h}$ after intravenous injection) and retention time (16 h). The use of $\mathrm{NEt}_{2} \mathrm{Br}_{2} \mathrm{BDP}$ in in vivo PDT was also explored using the same mouse model. Upon $808 \mathrm{~nm}$ laser irradiation, a dramatic decrease in tumor size was detected as a result of singlet oxygen-mediated necrosis of cancer cells in a lysosome-associated pathway. Additionally, $\mathrm{NEt}_{2} \mathrm{Br}_{2} \mathrm{BDP}$ enables tracking of the efficacy of PDT in a self-reporting manner by monitoring the decrease in fluorescence intensity in the tumor region after the therapeutic action takes place (Figure 3b, c).

Another pH-responsive BODIPY-based PDT system was developed by Wang and co-workers. In this construct, watersoluble pillar[5] arene was used as a host (carrier) for a diiodoBODIPY derivative that contains a quaternary ammonium tail. ${ }^{[25]}$ Supramolecular vesicles (pseudorotaxane) formed with pillar[5]arene and the modified dye are highly stable under physiological conditions but undergo disassembly in an acidic environment to release the dye. In a proof of principle study, the vesicles were found to be cytotoxic only to A549 cancer cells under $690 \mathrm{~nm}$ irradiation, suggesting that they disassemble a)

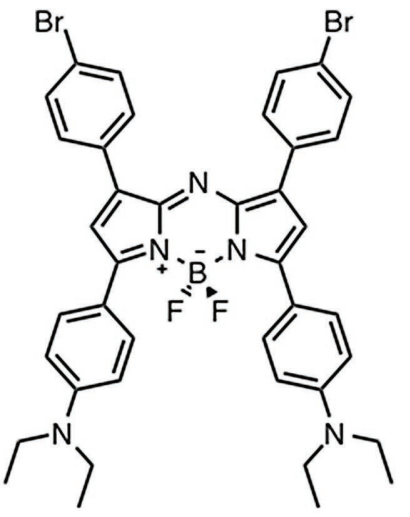

$\mathrm{NEt}_{2} \mathrm{Br}_{2} \mathrm{BDP}$
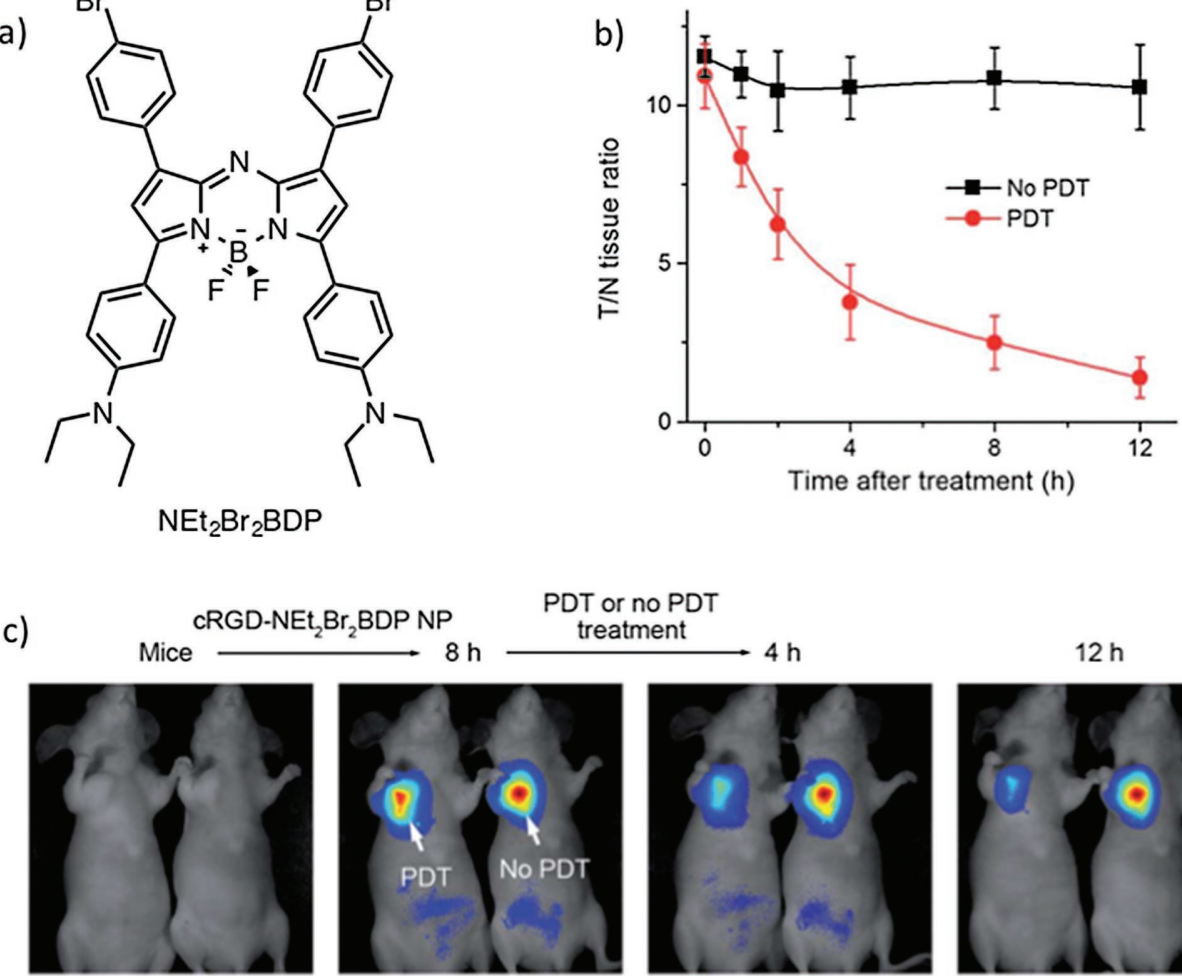

DT or no PDT

eatment
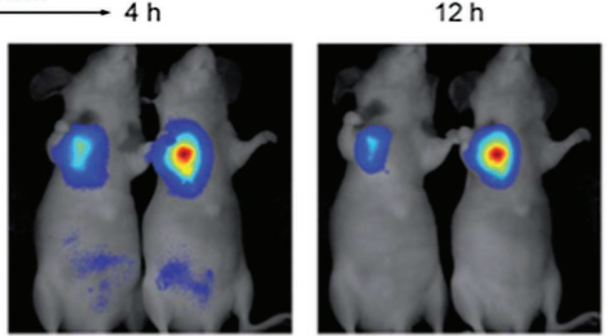

Figure 3. a) Structure of the $\mathrm{pH}$-activatable aza-BODIPY-based PS. b) $\mathrm{T} / \mathrm{N}$ tissue fluorescence ratios of U87MG tumor-bearing mice with respect to time before and after PDT treatment. c) In vivo PDT action and monitoring the therapeutic efficacy on U87MG tumor-bearing mice with CRGD$\mathrm{NEt}_{2} \mathrm{Br}_{2} \mathrm{BDP}$ NP (irradiation wavelength is $808 \mathrm{~nm}$ ). Adapted with permission. [24] Copyright 2015, Royal Society of Chemistry. 

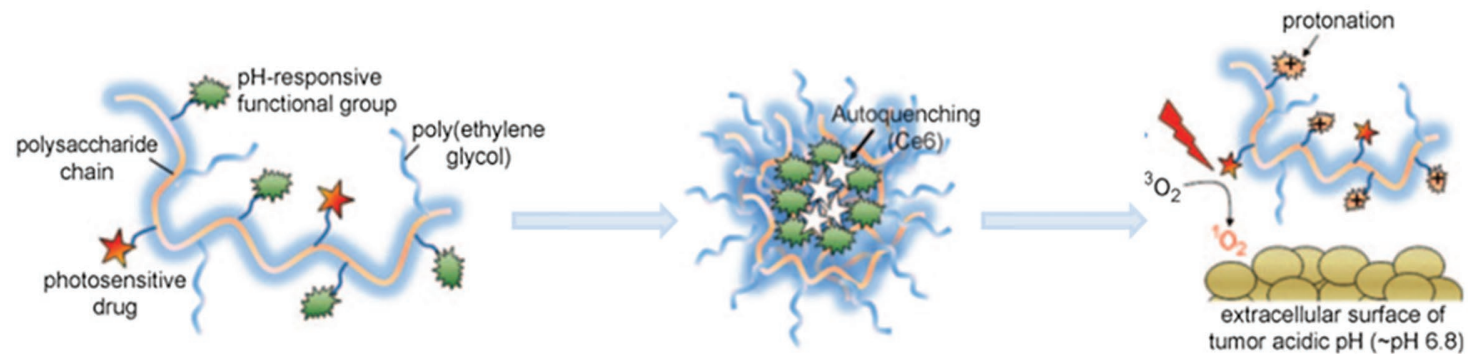

Figure 4. Schematic illustration of a polysaccharide/drug complex. This complex assembles and undergoes autoquenching efficiently at high pH, while in the acidic environment of tumor tissues protonation occurs and photoactivities restored, thereby destroying the tumor. Reproduced with permission. ${ }^{[26]}$ Copyright 2011, John Wiley and Sons.

under the slightly acidic conditions present in the cell to liberate the PDT-active diiodo-BODIPY derivative. Also, the vesicles can be simultaneously loaded with the well-known cancer chemotherapy drug doxorubicin (DOX). The viability of the A549 cancer cells was found to decrease upon treatment with the DOX-loaded vesicles in the absence of irradiation (dark toxicity), indicating that efficient release of DOX from the vesicle takes place. Finally, cell death is more pronounced when the cell treated, DOX-loaded vesicles are irradiated as a result of chemo-photodynamic dual therapeutic action.

In 2011, Lee et al. described a very interesting molecular "Trojan horse" composed of a polysaccharide/chlorine e6 conjugate (Figure 4) that selectively switches into an active complex for tumor destruction at $\mathrm{pH} 6.8 .^{[26]}$ This conjugate exists in a self-assembled, autoquenching, non-emissive, and non-PS active supramolecular form at high $\mathrm{pH}$. Owing to the change occurring in its surface charge when placed into an acidic environment, the complex is transformed to random molecules that are emissive and PDT active. Thus, this conjugate produced higher singlet oxygen at $\mathrm{pH} 6.8$ or $\mathrm{pH} 6.4$ than at $\mathrm{pH}$ 7.4. As a result, it showed higher phototoxicity against HeLa cells at acidic environment than at neutral condition. In vivo fluorescence imaging showed that the fluorescence signal in tumor tissues is fluctuating after treating with this conjugate. ${ }^{[26]}$

More recently, Liu et al. reported a $\mathrm{pH}$-responsive nanoaPS, which is composed of a complex of poly L-lysine (PLL), an AIEgen (tetraphenylsilole, TPS), an ACQ PS (pheophorbide $\mathrm{A}$, PheA), and PEG with a targeting group. ${ }^{[27]} \mathrm{At} \mathrm{pH} \mathrm{7.4,} \mathrm{the}$ AIEgen component displays green fluorescence, while emission from the PheA moiety is quenched. In contrast, at pH 5.0, the PheA group emits red fluorescence and serves as a singlet oxygen generator, while the AIEgen-related green fluorescence is diminished owing to protonation and disassembly of the complex. These properties make this nano-aPS a potential agent for specific bio-imaging, cancer therapy, and self-tracking. ${ }^{[27]}$

In the same year, Battogtokh and Ko used PheA as basis for a nano-PS as part of a folate-targeted, albumin-PheA conjugate (FA-BSA-c-PheA), which contains an acid-responsive linker (cisaconityl). ${ }^{[28]}$ Because FA has a high affinity for folate receptor that is overexpressed on the surface of tumor cells, the nanoaPS has effective tumor-targeting characteristics. Moreover, the phototoxicity of the nano-PS is activated upon cleavage of the acid-responsive linker at $\mathrm{pH}$ 5.0. It was described that the in vitro phototoxicity of this nano-PS is similar to free PheA, but its tumor accumulation is much better than that of free PheA. ${ }^{[28]}$
The first attempt to develop ZnPc-based aPSs was made in 2012 by Lo and co-workers, ${ }^{[29]}$ who prepared a self-quenching ZnPc dimer linked with an mild acid responsive ketal unit. The photoactivity of this aPS is activated by cleavage of the ketal unit at $\mathrm{pH}$ 5.0-6.5. This process results in separating the phthalocyanine moieties from each other, leading to an increase fluorescent emission efficiency and singlet oxygen generation. In fact, the quenching percentages for singlet oxygen production and fluorescence emission for this $\mathrm{ZnPc}$ dimer at $\mathrm{pH} 7.4$ are $66 \%$ and $84 \%$, respectively. Although reactivation of its photophysical properties at $\mathrm{pH}$ 5.0-6.5 occurs slowly, the ability of this system to generate singlet oxygen even in small amounts enables it to cause oxidative damage to cancer cells. Therefore, this $\mathrm{ZnPc}$ dimer is a cancer-specific $\mathrm{pH}$-responsive fluorescent probe and PS for targeted PDT. ${ }^{[2]}$

In 2015, Huang and co-workers specially designed and pre-

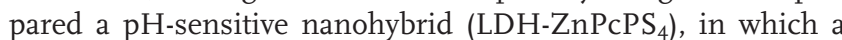
hydrophilic and negative $\mathrm{ZnPcPS}_{4}$ group and a cationic layered double hydroxide (LDH) are held together through electrostatic interactions (Figure 5). ${ }^{[30]}$ Pure $\mathrm{ZnPcPS}_{4}$ has excellent photophysical properties at $\mathrm{pH} 7.4$ and displays a maximum absorption wavelength around $692 \mathrm{~nm}$ at $\mathrm{pH}$ 7.4. However, its fluorescence emission and singlet oxygen generation ability are quenched by about $80 \%$ upon loading on LDH. While LDH$\mathrm{ZnPcPS}_{4}$ is stable under neutral conditions, slightly acidic conditions induce the release of $\mathrm{ZnPcPS}_{4}$ from LDH. As a result, the photoactivities of $\mathrm{ZnPcPS}_{4}$ are restored to about $60 \%$. Observations made in in vitro studies indicate that LDH$\mathrm{ZnPcPS}_{4}$ possesses a greatly enhanced photocytotoxicity against HepG2 cells with an $\mathrm{IC}_{50}$ value of $0.053 \mu \mathrm{M}$ as compared to that of pure $\mathrm{ZnPcPS}_{4}(1.27 \mu \mathrm{M})$. The strategy employed to design this aPS system should be applicable to the development of PSs that have specific tumor targeting abilities, highly activatable photocytotoxicities, and minimum side effects. ${ }^{[30]}$

Because of the strongly hydrophobic nature of their extended conjugated electronic cores, Pcs tend to aggregate in solution, a phenomenon that limits their applications. ${ }^{[31]}$ Ready modification of axial ligands reduces the aggregation propensity of PCs to some extent, so that SiPcs have better properties than do ZnPc. This reasoning led $\mathrm{Ng}$ et al. to develop several SiPc-based aPSs. ${ }^{[32-34]}$ The first one explored was a tetraamino-SiPc, which has greatly enhanced photoactivity efficiencies under highly acidic conditions. This feature makes tetraamino-SiPc a promising $\mathrm{pH}$-sensitive fluorescent probe and PS for PDT. ${ }^{[32]}$ 


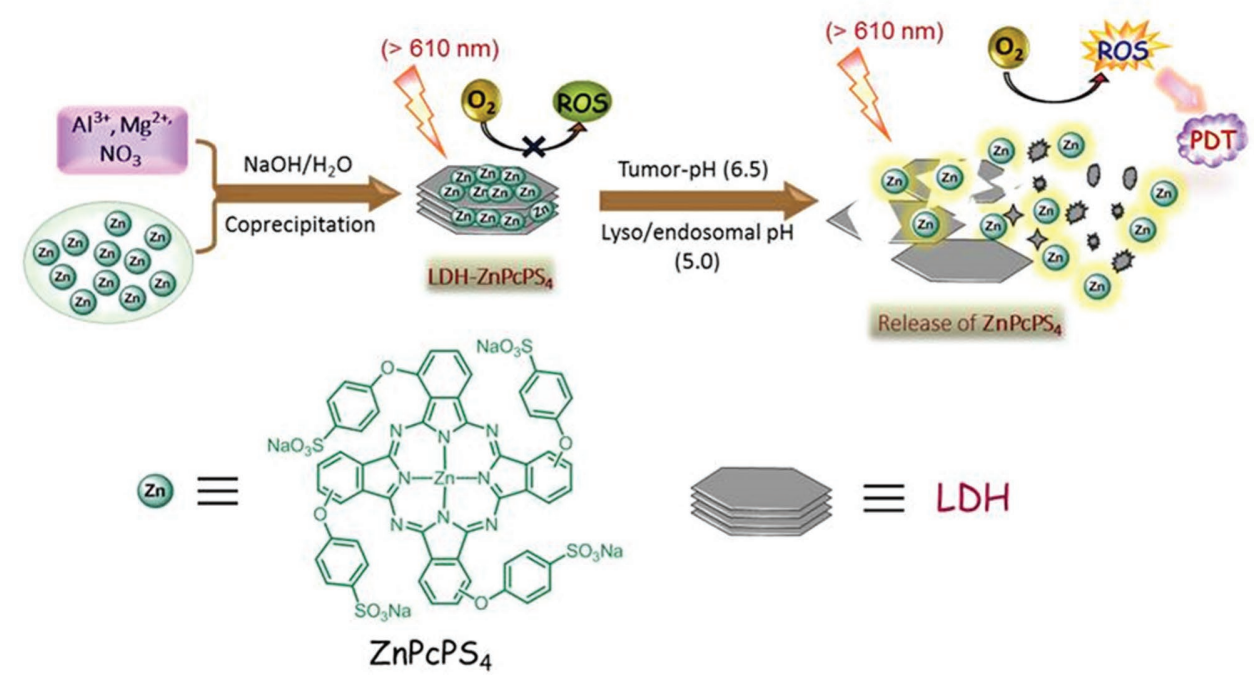

Figure 5. Illustration of the construction of a pH-sensitive nanohybrid $\left(\mathrm{LDH}-\mathrm{ZnPCPS}_{4}\right)$ and its proposed mechanism for activation in PDT associated with acid promoted release of $\mathrm{ZnPcPS}_{4}$ y. Reproduced with permission. ${ }^{[30]}$ Copyright 2015, John Wiley and Sons.

\subsection{Higher Intracellular GSH Concentrations in Cancer Cells Activates Specific Imaging and PDT}

Since the aforementioned aPSs are based on the activation of extracellular low $\mathrm{pH}$ in tumor tissue, they may be destructive to the tumor by first destroying the non-tumor cells or biomolecules in the extracellular matrix of tumor tissue. Intracellular stimuli-activated mechanism may be a more direct strategy to destroy the cancer cells. As we know, the concentration of intracellular glutathione (GSH) is about $10 \mathrm{~mm}$, which is much higher than it is in extracellular environments (ca. $2 \mu \mathrm{M}$ ). This feature can be utilized advantageously in strategies to activate specific intracellular drug release. ${ }^{[35]}$ Moreover, GSH levels in tumor tissues are usually even higher than those in normal tissues. ${ }^{[36]}$ Thus, approaches for tumor-targeted PS release and activation can also be based on reactions of GSH. Recently, a number of drug delivery systems and fluorescence probes that rely on biothiol-responsive protocols have been described. ${ }^{[37-41]}$ To the best of our knowledge, only two recent examples of this approach utilizing different PSs have been described.

$\mathrm{Wu}$ and co-workers designed caged PS and fluorescent reporter modules, which are covalently linked through a GSH responsive disulfide linker. ${ }^{[4]}$ The theranostic design of these types of substances takes advantage of the presence of two chromophores operating separately as an activatable PS and a fluorescent reporter. Consequently, singlet oxygen generation and fluorescence are not operating competitively in this system, which enables maximization of both outputs.

One of these types of dual active systems contains a 2,6-diiodo-BODIPY sensitizer moiety, which is inactive owing to singlet-excited state quenching through PET. At the same time, a mono-styryl BODIPY group serving as a reporter is almost non-emissive as a result of PET quenching of an internal 2,4-dinitrobenzenesulfonyl (DNBS) group that would have been capable of serving as an energy transfer donor. GSH, in high concentrations in cancer cells, promotes cleavage of the disulfide bond liberating the DNBS group, thus turning on its PS property $\left(\Phi_{\Delta}=0.72\right)$ and increasing the emission intensity of the reporter group $\left(\Phi_{\mathrm{F}}=0.48\right)$. This approach, in which both PDT and fluorescence monitoring properties are activated by GSH, was also shown to be applicable to HeLa cells.

In another recent approach, Turan et al. incorporated a DNBS quenching moiety into a dibromo-BODIPY core, which possesses triethyleneglycol units for improved water solubility (PS1) (Figure 6). ${ }^{[45}$ GSH-mediated activation of therapeutic action was found to take place in HCT116 cancer cells. Specifically, upon irradiation at $660 \mathrm{~nm}$, emission from FITC-Annexin-V labeled membranes is observed along with singlet oxygen induced apoptosis of the cells $\left(\mathrm{IC}_{50} \sim 0.02 \mu \mathrm{M}\right)$. It is worthy of note that singlet oxygen is not always quenched by the PET effect, which has been reported in many triplettriplet annihilation upconversion system. ${ }^{[46-48]}$ Our very recent research also indicates that some special phthalocyanines show fluorescence quenching induced by PET, while they still generate excellent singlet oxygen.

Heavy-atom free PSs have attracted great interest in recent years because they have minimal dark toxicity that arises from the presence of atoms such as bromine, iodine, and several lanthanides. Heavy-atom-induced dark toxicity is more pronounced when high concentrations of PSs are employed, a phenomenon that inherently restricts the applicable dosage of PSs and accordingly diminishes the efficacy of PDT. Because of this limitation, it is highly desirable to design PSs that have high ISC quantum yields but do not contain heavy atoms.

Kolemen et al. devised a GSH-activatable heavy-atom free PS based on an orthogonal BODIPY dimer scaffold. [21] The orthogonal arrangement of BODIPY moieties in this PS leads to a unique singlet excited state having in a doubly substituted tetraradical (DS-TR) electronic characteristic that facilitates ISC. Specifically, in this system two BODIPYs with similar electronic properties (symmetric BODIPY cores) are oriented orthogonally so that mixing of their $\pi$-systems does not occur. As a result, a degenerate pair of HOMOs and LUMOs exist, which is the key criterion for formation of DS-TR singlet excited state. ${ }^{[49]}$

Based on these results, an orthogonal dimer containing a reactive styrene double-bond was designed (5, Figure 7a), 


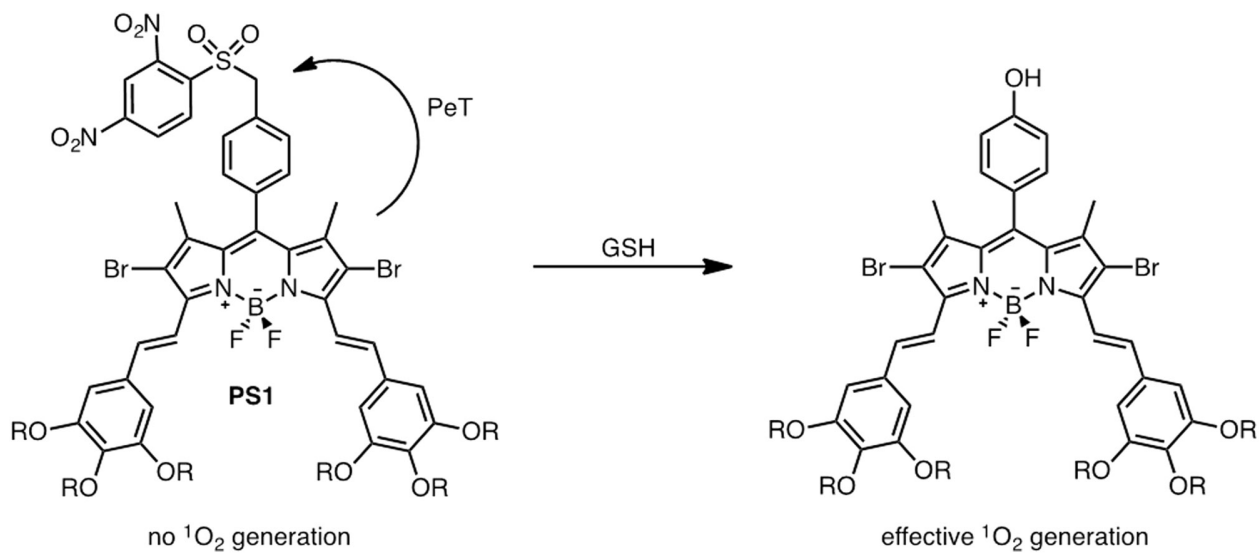

Figure 6. Structure of GSH-responsive PS1 bearing a DNBS group. Reproduced with permission. ${ }^{[45]}$ Copyright 2014, John Wiley and Sons.

in which one of the BODIPY core groups (BOD2) has an extended p-system (asymmetric dimer). This feature blocks formation of degenerate HOMOs and LUMOs and thus suppresses formation of the DS-TR state and consequent singlet oxygen generation. ${ }^{[50]}$ GSH in cancer cells reacts with the styryl double bond, disrupting the extended $\pi$-conjugation and isolating a methylpyridinium (MP) moiety from the orthogonal BODIPY dimer. Upon photoexcitation of the GSH adduct (5-GSH), through-space charge transfer takes place from BOD1 to electron-deficient MP, in which BOD2 behaves like an orthogonal spacer (Figure 7a). It is known that when orbitals responsible for charge transfer transitions are well separated and oriented in an orthogonal arrangement, the transition causes a change in orbital angular momentum, which is compensated by a spin angular momentum change.
This spin-state change favors ISC in the PS and promotes PDT action.

Singlet oxygen generation promoted by this aPS was demonstrated by using a singlet oxygen trap method and by monitoring its phosphorescence at $1270 \mathrm{~nm}$ with the help of easily prepared $\mathbf{5 r}$ ("always on") that is electronically similar to the 5-GSH adduct, which is the pre-activated analogue of 5 (Figure 7a). The actual activatable PS 5 was tested using both healthy and cancer cells. Red emission from Annexin V-AF594 dye, seen using confocal microscopy, clearly indicates that intracellular activation of $\mathbf{5}$ occurs and that singlet oxygen induced apoptosis of HeLa cells is promoted (Figure 7b). The survival rates for HeLa and SKHep 1 cancer cells treated in this manner were found to be around $40 \%$. On the other hand, non-cancerous NIH $3 \mathrm{~T} 3$ and WI38 VA13 cells have an approximate $80 \%$ survival rate under
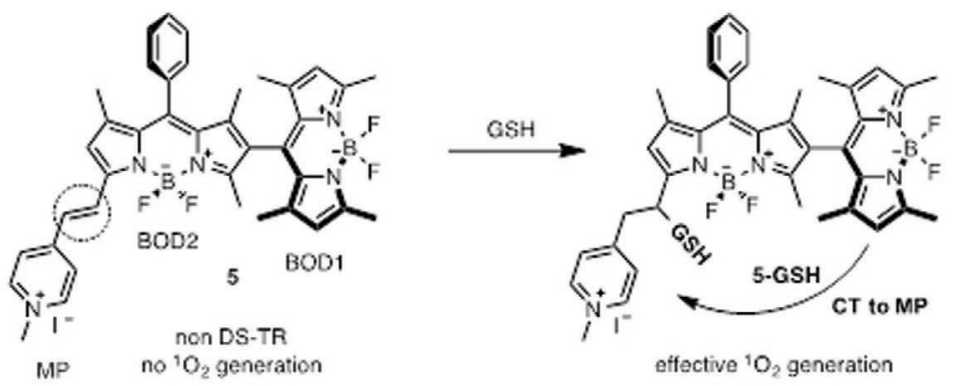

effective ${ }^{1} \mathrm{O}_{2}$ generation
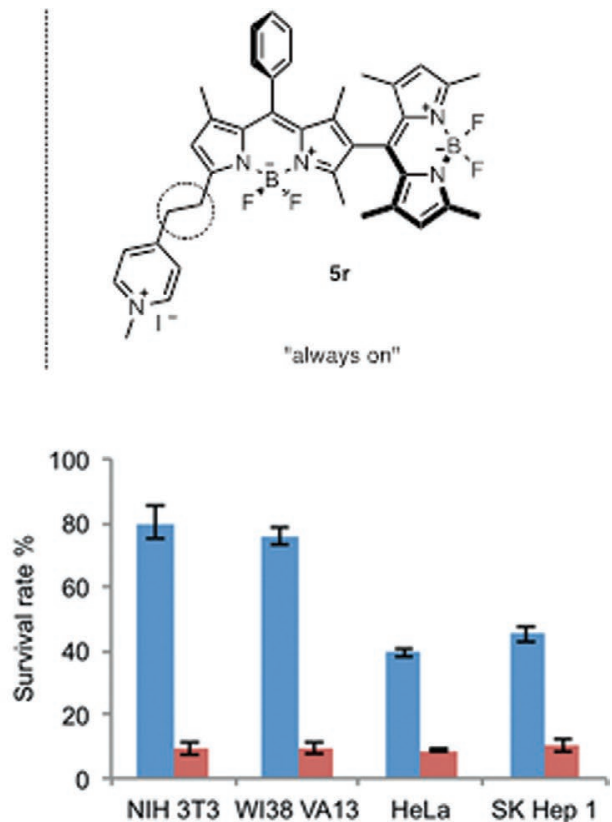

Figure 7. a) Reaction of activatable, heavy atom-free PS (5) with GSH. Structure of the pre-activated (always on) PS (5r). b) Confocal images of HeLa cells incubated with (5). Blue channel: DAPI, for nucleus staining, green channel: emission of the PS, red channel: Annexin V-AF594, an apoptosis marker. c) Survival rates of healthy cells (NIH 3T3, WI38 VA13) and of cancer cells (HeLa, SK Hep 1) which were incubated with (5) or (5r). Reproduced with permission. ${ }^{[50]}$ Copyright 2015, John Wiley and Sons. 


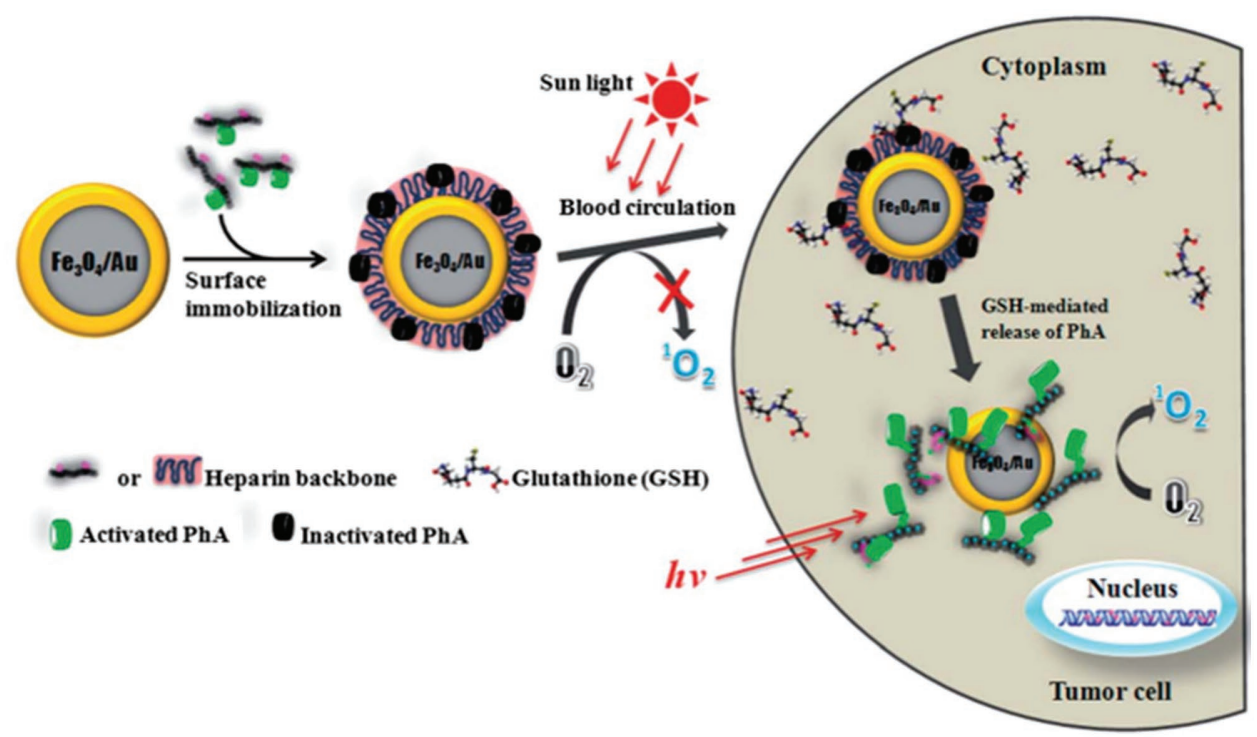

Figure 8. Schematic illustration of $\mathrm{GSH}$-responsive photoactivities of $\mathrm{Fe}_{3} \mathrm{O}_{4} / \mathrm{Au} / \mathrm{H}$-PhA nanohybrid for cancer-selective PDT. Reproduced with permission. ${ }^{[42]}$ Copyright 2014, Royal Society of Chemistry.

identical conditions. In addition, in the case of the "always on" positive control, PS $5 r$, cell viability was $10 \%$ for both healthy and cancer cells (Figure 7c). HeLa cells were also treated with buthioninesulfoxime (BSO), a known GSH synthesis inhibitor. The observation of a notable cell viability increase caused by this inhibitor demonstrates that GSH-mediated selective activation of the PS in cancer cells is taking place.

Huh et al. developed a nanohybrid containing a PS (PheA), an $\mathrm{Fe}_{3} \mathrm{O}_{4} / \mathrm{Au}$ nanoparticle and a polysaccharide heparin (Figure 8). ${ }^{[42]}$ In the hybrid, a thiolated PhA-heparin conjugate (H-PhA), serving as a supramolecular PS, is immobilized on the surfaces of $\mathrm{Fe}_{3} \mathrm{O}_{4} / \mathrm{Au}$ nanoparticles through gold-thiol bonds. The photoactivitiy of the PS is quenched by Au in the shell. Exchange of the thiol groups of the inactive PS on the Au surface with those of GSH present in cancer cells releases the photoactive PS. These observations suggest that this nanohybrid may be useful for tumor-selective PDT. ${ }^{[42]}$

Recently, Li et al. designed another GSH-responsive, targeted theranostic nanoparticle platform. ${ }^{[43]}$ In this system, chlorin e6 is conjugated with a matrix metalloproteinase-2 cleavable polypeptide and bonded to a polyethylene glycol via a GSH responsive disulfide linker. Interestingly, this substance self-assembles to form nanoparticles. In addition, unlike free chlorin e6, the cytotoxicity of the nanoparticles is not lost upon irradiation. The results of animal experiments showed that the nanoparticles have significantly enhanced tumor selectivity and an increased PDT efficiency. As a result, this smart nanoparticle aPS system holds great potential for use in tumor-targeting imaging and PDT. ${ }^{[43]}$

\subsection{Enzymes/Proteins Overexpressed in Cancer Cells Activate Specific Imaging and PDT}

Over the last several years, many studies have probed the use of enzymes to promote the photoactivities of porphyrin-based aPSs. Among these systems, which consist of a disease-targeted linker, a PS and a quencher, the "photodynamic molecular beacons" (PMB) developed by Zheng and co-workers are noteworthy. ${ }^{[51-61]}$ The excited state of the PS in this aPS is efficiently quenched via Förster Resonance Energy Transfer (FRET) to a quencher that is closely positioned through a linker. When the linker is cleaved by a cancer-specific stimulus, such as an enzyme, the quencher separates from the PS associated with regeneration of its photoactivity. Application of the PMB concept to the development of tumor-targeted PSs is very promising because it not only enables the design of systems that have tumor specific PS delivery capabilities but also has the ability to undergo controlled activation by a cancer specific biomarker. In addition, this concept can also be used to design other activation strategies to treat diseases other than cancer. ${ }^{[62]}$

In 2011, Zheng et al. described a new class of aPSs, called unquenched activatable photosensitizer (QUaPS), in which singlet oxygen generation is unquenched while fluorescence emission responds to a specific enzyme. ${ }^{[63]}$ In this system, the PS porphyrin pyropheophorbide (Pyro) is conjugated to 5-carboxyX-rhodamine (Rox) via a linker comprised of a caspase- 3 targeted peptide GDEVDGSGK (Figure 9). Rox serves as a donor in a FRET pair with the acceptor of Pyro. Interestingly, singlet oxygen production by this system is not quenched prior to caspase treatment but emission from the porphyrin is quenched by FRET. Consequently, upon Pyro excitation this QUaPS has enhanced photocytotoxicity against HT-29 cancer cells. The system can be used for ratiometric fluorescent imaging (with Rox excitation) of caspase-3 activation in cancer cells, following induction of cell death by Pyro excitation. Although having one recognized limitation of QUaPS, associated with detection interference caused by singlet oxygen induced Pyro self-bleaching, the method still holds great promise as novel feedback-oriented PDT strategy involving treating the targeted areas with an appropriate PS and light dose. ${ }^{[63]}$

Dong and co-workers recently described a protein-activated theranostic agent. ${ }^{[64]}$ This system is composed of nanoparticles 


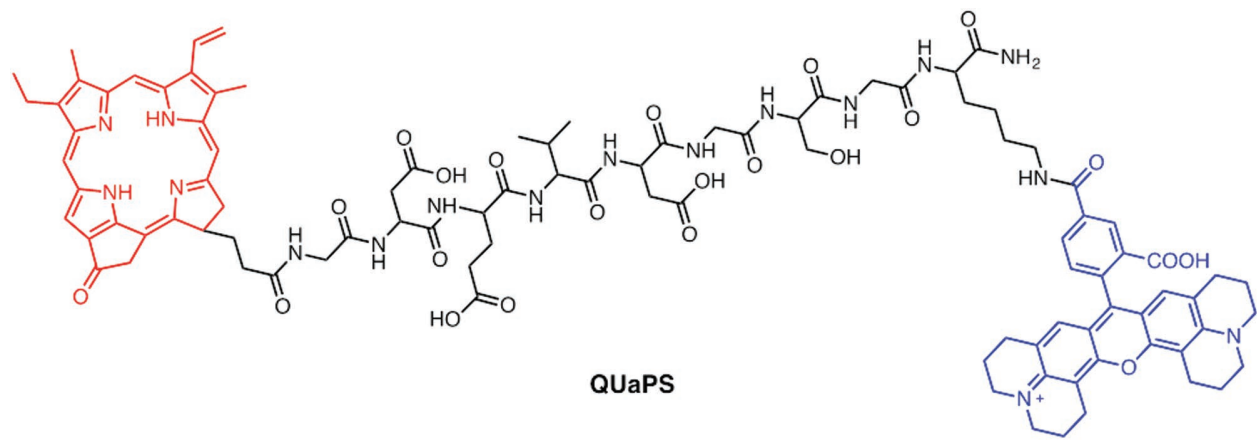

Figure 9. Structure of an aPS with a caspase-3 cleavable linker.

(DBHA-NPs) formed by conjugation of a heavy-atom decorated BODIPY with hyaluronic acid (HA) (Figure 10a), and it targets the HA receptor CD44, which is overexpressed in many cancer cells. The self-assembled, auto-quenching and spherical DBHA-NPs are disaggregated in HCT-116 cancer cells owing to overexpression of CD44. As a result, the nanoparticles display enhanced emission and they promote singlet oxygen generation. The behavior of this system was validated by its suppression of tumor growth in HCT-116 cancer cell bearing mouse models (Figure 10b). In A2780 cancer cells (low CD44 expression) the fluorescence intensity of the DBHA-NPs decrease gradually compared to that in HCT-116 cells proving that specific targeting takes place. It is concluded that uptake of these nanoparticles occurs selectively through CD44 receptor mediated endocytosis. In a report published last year, Choi et al. described a porphyrin-based aPS activated by cathepsin B. ${ }^{65]}$ In this system, chlorin e4 (Ce4) is conjugated with a closely posited folic acid (FA) group, which serves as a quencher, through a short peptide linker, which is cleaved by cathepsin B. This smart dual-selective theranostic agent becomes highly fluorescent and promotes photocytotoxicity only when the peptide linker is cleaved by cancer-associated cathepsin B in folate receptor-positive cancer cells. The results of both cell and animal studies indicate that this aPS is useful for dual-selective NIR fluorescent imaging and cancer-specific PDT.

Earlier, Nagano et al. developed an interesting enzymeresponsive aPSs based on a small molecule dye that is capable of selectively inducing photooxidative damage against $\beta$-galactosidaseexpressing cancer cells. ${ }^{[66,67]}$ This system, composed of a selenium-substituted rhodol scaffold modified with a $\beta$-galactoside (HMDESeR- $\beta \mathrm{Gal}$ ) cancer-targeting moiety, is not photocytotoxic due to the influence of galactoside moiety (Figure 11). The singlet oxygen generation capability of HMDESeR- $\beta \mathrm{Gal}$ is dramatically enhanced as a consequence of a structural change promoted by action of a cancer cell derived $\beta$-galactosidase. In a model study, these workers showed that when larval Drosophila melanogaster wing disks are treated with HMDESeR- $\beta$ Gal and irradiated, cell death takes place selectively in the posterior region where $\beta$-galactosidase is expressed. ${ }^{[66]}$ An example of a non-enzymatic protein acting as an effector for aPS is seen in studies by Jin et al. The targeting and triggered activatable system is composed of a folate-porphysome assembled from porphyrin-phospholipid conjugates. ${ }^{[68]}$ The folate receptor-targeting porphysome containing a FA-PEG moiety in the porphysome base displays nanostructureinduced super-quenching of photoactivities of the porphyrin $(>90 \%)$ as compared with the corresponding disassembled form of

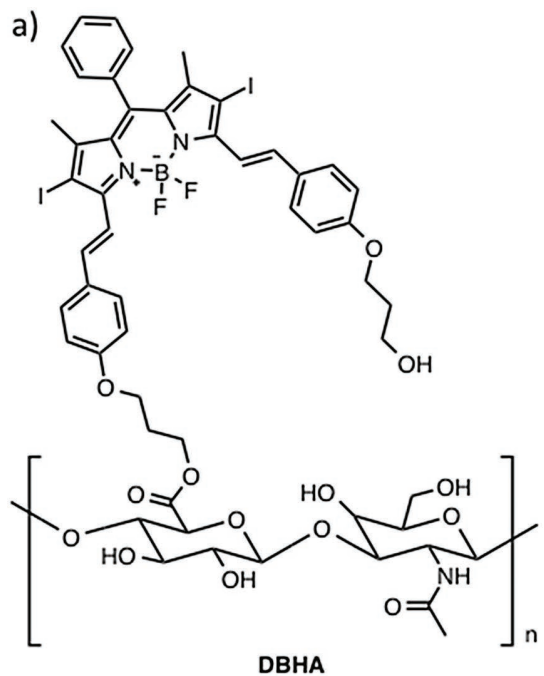

b)

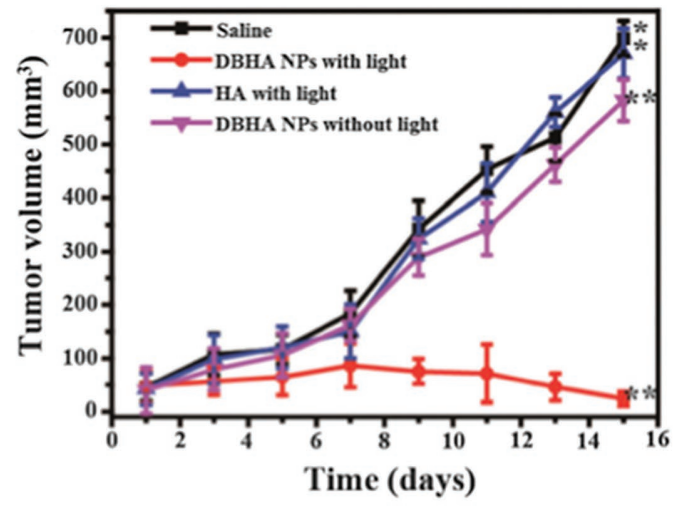

Figure 10. a) Enzyme-activated PS (DBHA-NPs). b) Time-dependent tumor volume change in the HCT-116 mice models in the presence of light or in the dark. Reproduced with permission. ${ }^{[64]}$ Copyright 2016, Royal Society of Chemistry. 


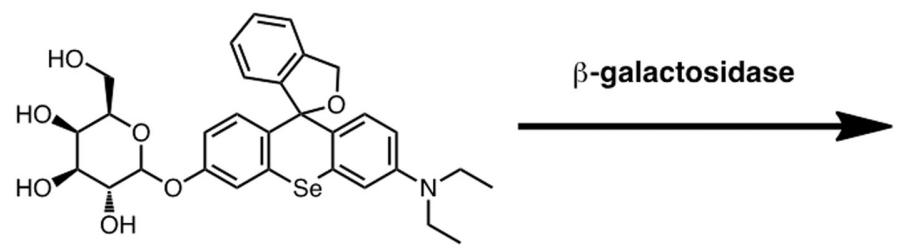

HMDESeR- $\beta$ Gal<smiles>CCN(CC)[N+](CC)=C1C=C2Oc3cc(Cl)ccc3C(c3ccccc3CO)=C2CC1</smiles>

HMDESeR

Figure 11. Structure of HMDESeR- $\beta$ Gal and $\beta$-galactosidase response.

the nanohybrid. FA facilitated cellular uptake of the nanoparticles into cancer cells leads to efficient disruption of the nanohybrids in the cells that blocks quenching of PS fluorescent emission and singlet oxygen production. In both cell and animal studies, FAporphysome were observed to have folate receptor-targeted PDT activity, which demonstrates the applicability of targeting-responsive PDT reactivation of porphysome for cancer-specific therapy. ${ }^{[68]}$

Several non-enzymatic proteins are overexpressed in both tumor and non-tumor cells in cancer tissues. For example, the biotin receptor is overexpressed to a greater extent than the folate receptor in a large number of cancer cell lines. ${ }^{[69-71]}$ Tumor-associated macrophages (TAMs) highly express scavenger receptor-A (SR-A). ${ }^{[72,73]}$ Vascular endothelial growth factor receptor (VEGFR) and $\alpha_{v} \beta_{3}$-integrin also represent excellent targets for specific PS delivery systems. ${ }^{\text {[4-77] }}$ Thus, many potential approaches exist to construct special aPSs for bioimaging and targeted PDT based on the non-enzymatic protein stimuli.

\subsection{Other Activation Methods}

Besides those based on protein, $\mathrm{pH}$, and thiol responses, other mechanisms can be employed to stimulate aPSs. For example, in 2014 Choi et al. described a novel theranostic agent for specific imaging and therapy of the atherosclerotic lesions. ${ }^{[78]}$ The system is comprised of macrophage-targeted theranostic nanoparticles (MacTNPs) formed by conjugating Ce6 to hyaluronic acid. Fluorescence and singlet oxygen generation by MacTNPs in the native state is inhibited by a self-quenching effect between the conjugated PSs. Upon internalization of MacTNPs into the activated macrophages, excess reactive oxygen species (ROS) in the cells degrades the nanoparticles by cleaving the hyaluronic acid group (Figure 12). As a result, the released PS induces fluorescence emission and singlet oxygen generation. Particularly interesting is the fact that MacTNPs show enhanced photocytotoxicity against activated macrophage cells as compared with non-activated counterparts and human dermal fibroblasts. The ROS-sensitive MacTNPs, which have an excellent target-to-background ratio, may have high potential for use in specific NIR fluorescent imaging and in efficient PDT of atherosclerosis with minimal side effects. ${ }^{[78]}$ One year later, Ma et al. developed a very interesting new aPS, two-dimensional molecular beacon, that could be activated by
mRNA. They obtained good efficiency of cancer imaging and PDT by using this new aPS as a theranostic platform. ${ }^{[79]}$

\subsection{Dual-Responsive aPSs}

In 2009, Ozlem and Akkaya suggested that incorporating two control elements into a responsive PS would make singlet oxygen generation even more cell selective. ${ }^{[80]}$ In this approach, the maximum rate of singlet oxygen generation promoted by the PS would be reached only when two cancer related parameters are above threshold values, in a manner that resembles an AND logic gate. This concept was tested using a dual responsive aPS that is responsive to both acidity and $\mathrm{Na}^{+}$concentration. In 2014, Ng et al. developed a $\mathrm{pH}$ and thiol dual-responsive aPS for PDT. ${ }^{[34]}$ This system consists of a SiPc core that is linked to two ferrocene quencher groups through a hydrazone and disulfide responsive bridges (Figure 13). These linkages are cleaved under mildly acidic ( $\mathrm{pH}=4.5-6.8)$ conditions and by biothiols, respectively. Thus, in the slightly acidic and biothiol rich environment of cancer cells cleavage results in separation of the ferrocene moieties. As a result, this aPS displays a much higher photocytotoxicity to tumor tissues as compared to normal tissues. ${ }^{[34]}$

Kim et al. designed a $\mathrm{pH}$ and temperature dual-responsive lipopolymer hybrid system (Ce6@VNS) containing Ce6, phosphatidylcholine (PC), the pH-responsive phosphatidylethanolamine (PE)-p(His) conjugate, along with the temperature responsive FA conjugated PE-p(NIPAM). ${ }^{[1]}$ Self-assembly upon mixing these components creates nanospheres, which have a

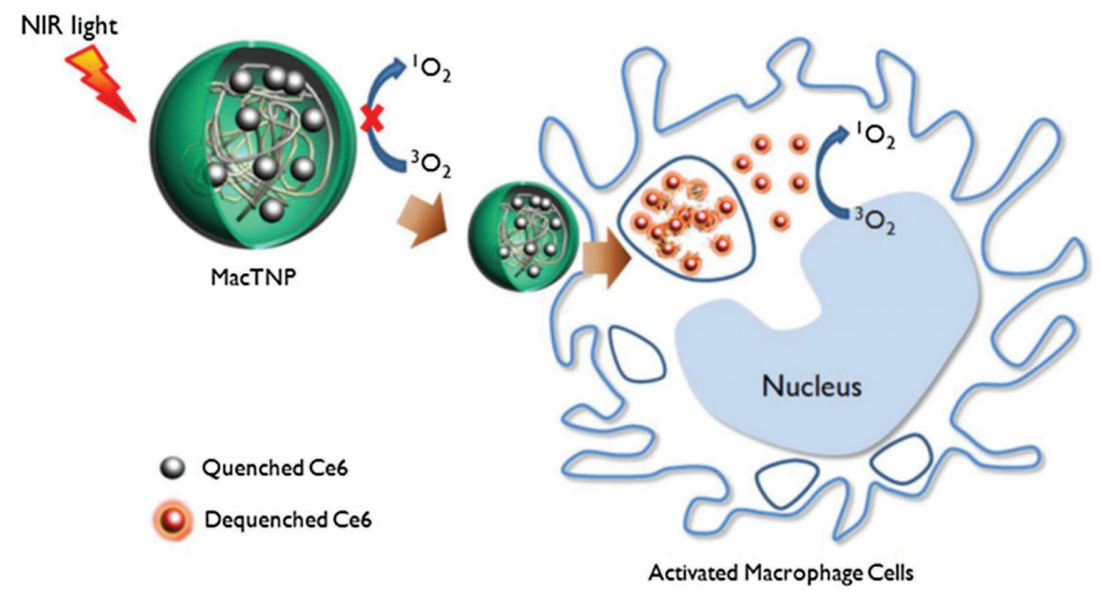

Figure 12. A schematic illustration of activatable agent for macrophage-targeted fluorescence imaging and subsequent PDT. Reproduced with permission. ${ }^{[78]}$ Copyright 2014, the Authors. 


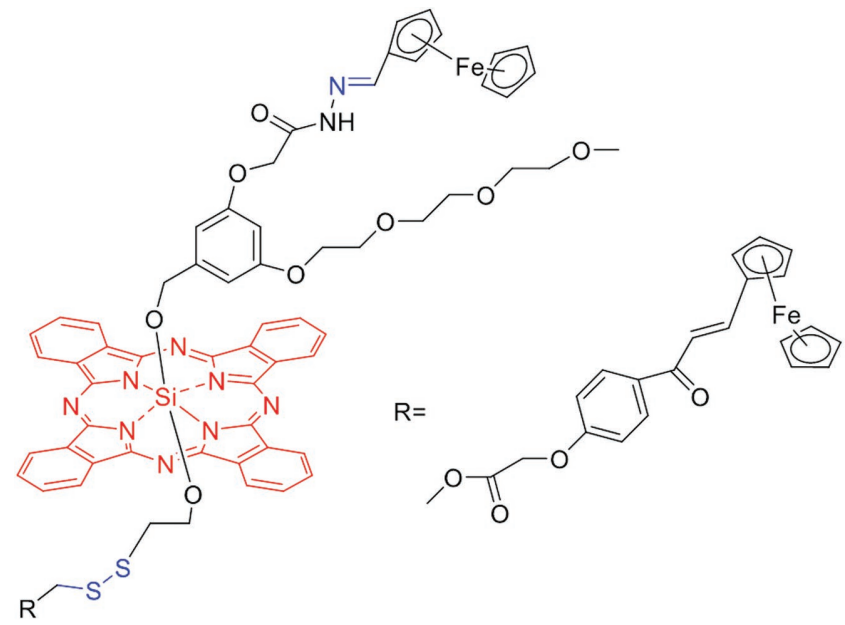

Figure 13. Structure of the "pH and thiol" dual-responsive unsymmetrical bisferrocenyl SiPc.

$\mathrm{p}$ (His) group sensitive to $\mathrm{pH}$ and a $\mathrm{p}$ (NIPAM) moiety responsive to temperature. Compared to free Ce6, Ce6@VNS has a significantly enhanced photocytotoxicity against targeted KB cells. ${ }^{[81]}$

In a more recent report, Torres et al. also described a $\mathrm{pH}$ and thiol dual-responsive aPS that contains a pyrene-modified SiPc group, which should attract great interest as a multifunctional theranostic agent. ${ }^{[82]}$

Recently, Liu et al. described a series of special fluorophores that possess aggregation-induced emission (AIE) characteristics that can be used advantageously in the design of new aPSs. ${ }^{[83-85]}$ One system is a dual-selective enzyme-responsive bioprobe containing a PS for cancer-specific imaging and activatable PDT. ${ }^{[3]}$ The probe is composed of four parts including an AIE fluorogen for imaging and PS, a GFLG peptide with cathepsin B responsive properties, a specific tri-Asp linker for improved hydrophilicity, and a cRGD-targeting group. In aqueous media, the photoactivities of the probe are quenched by the absence of exciton energy induced by intramolecular motions. However, fluorescence emission and phototoxicity of the probe are activated following internalization into cancer cells owing to cleavage of the GFLG group by cathepsin B. Efficient fluorescence emission and phototoxicity is only obtained in the aggregate model induced by the tumor-related reactivation. ${ }^{[83]}$ This system serves as a prototype of a design for constructing aPSs that are not composed of quenchers/scavengers or energy acceptors.

\section{Conclusion and Outlook}

The field of PDT is at a stage where new approaches to design fluorescence imaging and singlet-oxygen-producing PSs are in demand. For a long time, the major activity within the area was limited to the synthesis and evaluation of new dyes as potential PSs. However, within the last decade, the focus of this activity has shifted and new strategies have been devised for PDT. For example, recent efforts have led to the development of aPSs, in which excited state processes are judiciously manipulated in response to cancer cell specific stimuli. As a result, "off-on" switching of singlet oxygen generation in response to cancer- related parameters has become a very promising approach to design new PDT agents. Also, advances have been made in constructing systems in which fluorescence imaging is possible at the site of singlet oxygen production, making the PSs selfcontained theranostic agents.

As previously mentioned, different activation strategies based on the anomalies of tumor have been designed and constructed. However, we can safely speculate that even more interesting and potentially useful aPSs are likely to be developed according to novel activation mechanisms, which utilize some other cancer-related parameters such as hypoxia, specific metal ions, other over-expressed proteins in tumors, and so on. In addition, to the best of our knowledge, most of the existing acidity-responsive $\mathrm{PSs}$ are activated at $\mathrm{pH}<6.5$, which does not fall into the region of tumor $\mathrm{pH}$. Thus, more attention should be paid to the more efficient and tumor-pHactivated PSs. As the limiting factors governing PDT become better understood and addressed, we can expect that the clinical utility for this treatment modality will grow. It is clear from a current perspective that the introduction of the aPS concept has contributed greatly to this field by enabling the design of smarter therapeutic agents and even rudimentary information processing systems targeting cancer.

\section{Acknowledgements}

X.L. and S.K. contributed equally to this work. JY is funded by a grant from the National Creative Research Initiative programs of the National Research Foundation of Korea (NRF) funded by the Korean government (MSIP) (No. 2012R1A3A2048814). EUA acknowledges support from Scientific and Technological Research Council of Turkey (TUBITAK; KBAG-102T480).

Received: August 7, 2016

Revised: September 29, 2016

Published online: December 9, 2016

[1] P. Agostinis, K. Berg, K. A. Cengel, T. H. Foster, A. W. Girotti, S. O. Gollnick, S. M. Hahn, M. R. Hamblin, A. Juzeniene, D. Kessel, M. Korbelik, J. Moan, P. Mroz, D. Nowis, J. Piette, B. C. Wilson, J. Golab, CA Cancer J. Clin. 2011, 61, 380.

[2] A. P. Castano, P. Mroz, M. R. Hamblin, Nat. Rev. Cancer 2006, 6, 535.

[3] B. C. Wilson, M. S. Patterson, Phys. Med. Biol. 2008, 53, R61.

[4] J. F. Lovell, T. W. B. Liu, J. Chen, G. Zheng, Chem. Rev. 2010, 110, 2839.

[5] P. Majumadar, R. Nomula, J. Zhao, J. Mater. Chem. C 2014, 2, 5982.

[6] V. Kral, J. Kralova, R. Kaplanek, T. Briza, P. Martasek, Physiol. Res. 2006, 55(Suppl), S3.

[7] M. J. Garland, C. M. Cassidy, D. Woolfson, R. F. Donnelly, Future Med. Chem. 2009, 1, 667.

[8] M. Ethirajan, Y. Chen, P. Joshi, R. K. Pandey, Chem. Soc. Rev. 2011, 40, 340 .

[9] A. Braun, J. Tcherniac, Ber. Dtsch. Chem. Ges. 1907, 40, 2709.

[10] C. C. Leznoff, A. B. P. Lever, Phthalocyanines, Properties and Applications; Vol. 1, Wiley, VCH: New York 1989.

[11] L. B. Josefsen, R. W. Boyle, Br. J. Pharmacol. 2008, 154, 1.

[12] N. Sekkat, H. v. d. Bergh, T. Nyokong, N. Lange, Molecules 2011, 17, 98.

[13] B. Roeder, D. Naether, T. Lewald, M. Braune, C. Nowak, W. Freyer, Biophys. Chem. 1990, 35, 303. 
[14] S. Singh, A. Aggarwal, N. V. S. D. K. Bhupathiraju, G. Arianna, K. Tiwari, C. M. Drain, Chem. Rev. 2015, 115, 10261.

[15] H. Ali, J. E. van Lier, Chem. Rev. 1999, 99, 2379.

[16] N. Boens, V. Leen, W. Dehaen, Chem. Soc. Rev. 2012, 41, 1130.

[17] Y. Ni, J. Wu, Org. Biomol. Chem. 2014, 12, 3774.

[18] T. Kowada, H. Maeda, K. Kikuchi, Chem. Soc. Rev. 2015, 44, 4953.

[19] S. G. Awuah, Y. You, RSC Adv. 2012, 11169.

[20] A. Kamkaew, S. H. Lim, H. Bulee, L. V. Kiew, L. Y. Chung, K. Burgess, Chem. Soc. Rev. 2013, 42, 77.

[21] Y. Cakmak, S. Kolemen, S. Duman, G. Y. Dede, Y. Dolen, B. Kilic, Z. Kostereli, L. Tatar-Yildirim, A. L. Dogan, D. Guc, E. U. Akkaya, Angew. Chem., Int. Ed. 2011, 50, 11937.

[22] L. E. Gerweck, K. Seetharaman, Cancer Res. 1996, 56, 1194.

[23] P. Montcourrier, I. Silver, R. Farnoud, I. Bird, H. Rochefort, Clin. Exp. Metastasis 1997, 15, 382.

[24] J. Tian, J. Zhou, Z. Shen, L. Ding, J.-S. Yu, H. Ju, Chem. Sci. 2015, 6, 5969.

[25] L.-B. Meng, W. Zhang, D. Li, Y. Li, X.-Y. Hu, L. Wang, G. Li, Chem. Commun. 2015, 51, 14381.

[26] S. Y. Park, H. J. Baik, Y. T. Oh, K. T. Oh, Y. S. Youn, E. S. Lee, Angew. Chem., Int. Ed. 2011, 50, 1644.

[27] Y. Yuan, R. T. K. Kwok, B. Z. Tang, B. Liu, Small 2015, 11, 4682.

[28] G. Battogtok, Y. T. Ko, J. Mater. Chem. B 2015, 3, 9349.

[29] M. R. Ke, D. K. P. Ng, P. C. Lo, Chem. Commun. 2012, 48, 9065.

[30] X. S. Li, M. R. Ke, W. Huang, C. H. Ye, J. D. Huang, Chem. Eur. J. 2015, 21, 3310.

[31] J. R. Darwent, P. Douglas, A. Harriman, G. Porter, M. C. Richoux, Coord. Chem. Rev. 1982, 44, 83.

[32] X. J. Jiang, P. C. Lo, S. L. Yeung, W. P. Fong, D. K. P. Ng, Chem. Commun. 2010, 46, 3188

[33] J. T. F. Lau, X. J. Jiang, D. K. P. Ng, P. C. Lo, Chem. Commun. 2013, 49, 4274.

[34] J. T. F. Lau, P. C. Lo, X. J. Jiang, Q. Wang, D. K. P. Ng, J. Med. Chem. 2014, 57, 4088.

[35] R. Cheng, F. Feng, F. Meng, C. Deng, J. Feijen, Z. Zhong, J. Controlled Release 2011, 152, 2.

[36] M. P. Gamcsik, M. S. Kasibhatla, S. D. Teeter, O. M. Colvin, Biomarkers 2012, 17, 671

[37] J. Lai, B. P. Shah, E. Garfunkel, K. B. Lee, ACS Nano 2013, 7, 2741.

[38] M. H. Lee, J. Y. Kim, J. H. Han, S. Bhuniya, J. L. Sessler, C. Kang, J. S. Kim, J. Am. Chem. Soc. 2012, 134, 12668.

[39] M. H. Lee, J. H. Han, P. S. Kwon, S. Bhuniya, J. Y. Kim, J. L. Sessler, C. Kang, J. S. Kim, J. Am. Chem. Soc. 2012, 134, 1316.

[40] L. Y. Niu, Y. S. Guan, Y. Z. Chen, L. Z. Wu, C. H. Tung, Q. Z. Yang, J. Am. Chem. Soc. 2012, 134, 18928.

[41] K. Xu, M. Qiang, W. Gao, R. Su, N. Li, Y. Gao, Y. Xie, F. Kong, B. Tang, Chem. Sci. 2013, 4, 1079.

[42] L. Li, M. Nurunnabi, M. Nafiujjaman, Y. Y. Jeong, Y. K. Lee, K. M. Huh, J. Mater. Chem. B 2014, 2, 2929.

[43] W. Hou, F. Xia, C. S. Alves, X. Qian, Y. Yang, D. Cui, ACS Appl. Mater. Interfaces 2016, 8, 1447.

[44] J. Zhao, L. Huang, X. Cui, S. Li, H. Wu, J. Mater. Chem. B 2015, 3, 9194.

[45] I. Simsek-Turan, F. Pir-Cakmak, D. C. Yildirim, R. Cetin-Atalay, E. U. Akkaya, Chem. Eur. J. 2014, 20, 16088.

[46] Z. Mahmood, J. Zhao, J. Org. Chem. 2016, 81, 587.

[47] C. Zhang, J. Zhao, X. Cui, X. Wu, J. Org. Chem. 2015, 80, 5674.

[48] K. Xu, J. Zhao, X. Cui, J. Ma, J. Phys. Chem. A 2015, 119, 468.

[49] S. Duman, Y. Cakmak, S. Kolemen, E. U. Akkaya, Y. Dede, J. Org. Chem. 2012, 77, 4516

[50] S. Kolemen, M. Işık, G. M. Kim, D. Kim, H. Geng, M. Buyuktemiz, T. Karatas, X.-F. Zhang, Y. Dede, J. Yoon, E. U. Akkaya, Angew. Chem., Int. Ed. 2015, 54, 5340.
[51] J. Chen, K. Stefflova, M. J. Niedre, B. C. Wilson, B. Chance, J. D. Glickson, G. Zheng, J. Am. Chem. Soc. 2004, 126, 11450.

[52] J. Chen, M. Jarvi, P. Lo, K. Stefflova, B. C. Wilson, G. Zheng, Photochem. Photobiol. Sci. 2007, 6, 1311.

[53] J. Chen, K. Stefflova, M. Warren, J. Bu, B. C. Wilson, G. Zheng, Proc. SPIE-Int. Soc. Opt. Eng. 2007, 6449, 1.

[54] J. Chen, P. Lo, M. Warren, K. Stffova, J. D. Cheng, B. C. Wilson, G. Zheng, Proc. SPIE 2007, 6626, 1.

[55] G. Zheng, J. Chen, K. Stefflova, M. Jarvi, H. Li, B. C. Wilson, Proc. Natl. Acad. Sci. U. S. A. 2007, 104, 8989.

[56] J. Chen, J. F. Lovell, P. Lo, K. Stefflova, M. Niedre, B. C. Wilsona, G. Zheng, Photochem. Photobiol. Sci. 2008, 7, 775.

[57] P. Lo, J. Chen, K. Stefflova, M. S. Warren, R. Navab, B. Bandarchi, S. Mullins, M. Tsao, J. D. Cheng, G. Zheng, J. Med. Chem. 2009, 52, 358.

[58] J. Chen, T. W. B. Liu, P. Lo, B. C. Wilson, G. Zheng, Bioconjugate Chem. 2009, 20, 1836

[59] T. W. Liu, M. K. Akens, J. Chen, L. Wise-Milestone, B. C. Wilson, G. Zheng, Bioconjugate Chem. 2011, 22, 1021.

[60] T. W. B. Liu, J. Chen, G. Zheng, Amino Acids 2011, 41, 1123.

[61] Q. Lin, J. Chen, Z. Zhang, G. Zheng, Nanomedicine 2014, 9, 105.

[62] J. P. Celli, B. Q. Spring, I. Rizvi, C. L. Evans, K. S. Samkoe, S. Verma, B. W. Pogue, T. Hasan, Chem. Rev. 2010, 110, 2795.

[63] J. F. Lovell, M. W. Chan, Q. Qi, J. Chen, G. Zheng, J. Am. Chem. Soc. 2011, 133, 18580.

[64] H. Shi, W. Sun, C. Liu, G. Gu, B. Ma, W. Si, N. Fu, Q. Zhang, W. Huang, X. Dong, J. Chem. Mater. B 2016, 4, 113.

[65] J. Kim, C. H. Tung, Y. Choi, Chem. Commun. 2014, 50, 10600.

[66] Y. Ichikawa, M. Kamiya, F. Obata, M. Miura, T. Terai, T. Komatsu, T. Ueno, K. Hanaoka, T. Nagano, Y. Urano, Angew. Chem., Int. Ed. 2014, 53, 6772.

[67] T. Yogo, Y. Urano, M. Kamiya, K. Sano, T. Nagano, Bioorg. Med. Chem. Lett. 2010, 20, 4320.

[68] C. S. Jin, L. Cui, F. Wang, J. Chen, G. Zheng, Adv. Healthcare Mater. 2014, 3, 1240.

[69] C. P. Leamon, Curr. Opin. Invest. Drugs 2008, 9, 1277.

[70] G. Russell-Jones, K. McTavish, J. McEwan, J. Rice, D. Nowotnik, J. Inorg. Biochem. 2004, 98, 1625.

[71] Y. Lu, P. S. Low, Adv. Drug Delivery Rev. 2002, 43, 675.

[72] A. Mantovani, S. Sozzani, M. Locati, P. Allavena, A. Sica, Trends Immunol. 2002, 23, 549 .

[73] T. Hagemann, J. Wilson, F. Burke, H. Kulbe, N. F. Li, A. Pluddemann, K. Charles, S. Gordon, F. R. Balkwill, J. Immunol. 2006, 176, 5023.

[74] S. Kumar, C. Li, Trends Immunol. 2001, 22, 129.

[75] P. Carmeliet, Nat. Med. 2003, 9, 653.

[76] W. M. Sharman, J. E. van Lier, C. M. Allen, Adv. Drug Delivery Rev. 2004, 56, 53

[77] A. M. Bugaj, Photochem. Photobiol. Sci. 2011, 10, 1097.

[78] H. Kim, Y. Kim, I. H. Kim, K. Kim, Y. Choi, Theranostics 2014, 4, 1

[79] D. Wu, G. Song, Z. Li, T. Zhang, W. Wei, M. Chen, X. He, N. Ma, Chem. Sci. 2015, 6, 3839

[80] S. Ozlem, E. U. Akkaya, J. Am. Chem. Soc. 2009, 131, 48.

[81] J. V. John, C. W. Chung, R. P. Johnson, Y. I. Jeong, K. D. Chung, D. H. Kang, H. Suh, H. Chen, I. Kim, Biomacromolecules 2016, 17, 20.

[82] E. van de Winckel, R. J. Schneider, A. de la Escosura, T. Torres, Chem. Eur. J. 2015, 21, 18551.

[83] Y. Yuan, C. J. Zhang, M. Gao, R. Zhang, B. Z. Tang, B. Liu, Angew. Chem., Int. Ed. 2015, 54, 1780.

[84] Y. Yuan, C. J. Zhang, R. T. K. Kwok, S. Xu, R. Zhang, J. Wu, B. Z. Tang, B. Liu, Adv. Funct. Mater. 2015, 25, 6586.

[85] Y. Yuan, S. Xu, C. J. Zhang, R. Zhang, B. Liu, J. Mater. Chem. B 2016, $4,169$. 